\title{
Engineering section analysis based on automatic feature points matching in reverse engineering
}

\author{
Lingjie SHU \\ 1.School of Computer Science and Software Engineering, East China Normal University, Shanghai , \\ China
}

Keywords: reverse engineering; feature point matching; section analysis; automation; product design

\begin{abstract}
As large scale complex surface product encounters problems such as large point cloud data density, various redundancy points and heavy processing tasks in reverse engineering, the Thesis analyzes necessity of data pre-processing in reverse engineering. For dispersing of data measurement, coarse point cloud becomes smooth and level through point cloud pretreatment and there are only a few miscellaneous points and the gained point cloud reduces operating difficulty of three-dimensional model reconstitution significantly; the Thesis proposes final judgment criterion $\Delta\left(s, s^{\prime}\right)$ during point cloud sampling. The judgment criterion meets requirement of high precision, fast speed and suitable simple principles and it can also reach the given maximum permissible error or quantity of the specified points and it applies to occasions having high requirement on geometric accuracy of reconstruction; at last, the Thesis takes certain large scale hydraulic turbine blade as an example and conducts sampling and reverse design modeling accuracy analysis and proves validity and feasibility of three-dimensional optical detection point cloud sampling method.
\end{abstract}

\section{Introduction}

Data acquisition is the foundation of point cloud treatment in reverse engineering. High efficiency and high precision acquisition of geometrical characteristic data of samples is an important research content in reverse engineering. Unlike tradition measurement means, surface scanning technology is optical non-contact measurement and will not be influenced by complexity of work-piece surface and can gain large quantity of data information in short time so as to provide original data for reverse design and three-dimensional quality detection promptly. Three-dimensional optical measurement technology is widely applied in various fields due to these advantages. However, density of data gained by the equipment is very huge and is influenced by working scope and complexity of the measured piece significantly during measurement. There are many redundancy points generally after data of multiple view is translated into the same coordinate system. Mass data points are redundant for surface reconstruction and quality detection and it also brings inconvenience for data storage and follow-up treatment. Therefore, sampling of scanning data with qualified precision is important work for reverse treatment and three-dimensional detection.

\section{Point cloud pretreatment technology}

Point cloud pretreatment procedure

According to Fig.1, point cloud pretreatment technology procedure includes acquisition of large quantity of point cloud data, point cloud pretreatment and surface reconstruction. Pretreatment technology is the core and difficulty of the research and it includes: 


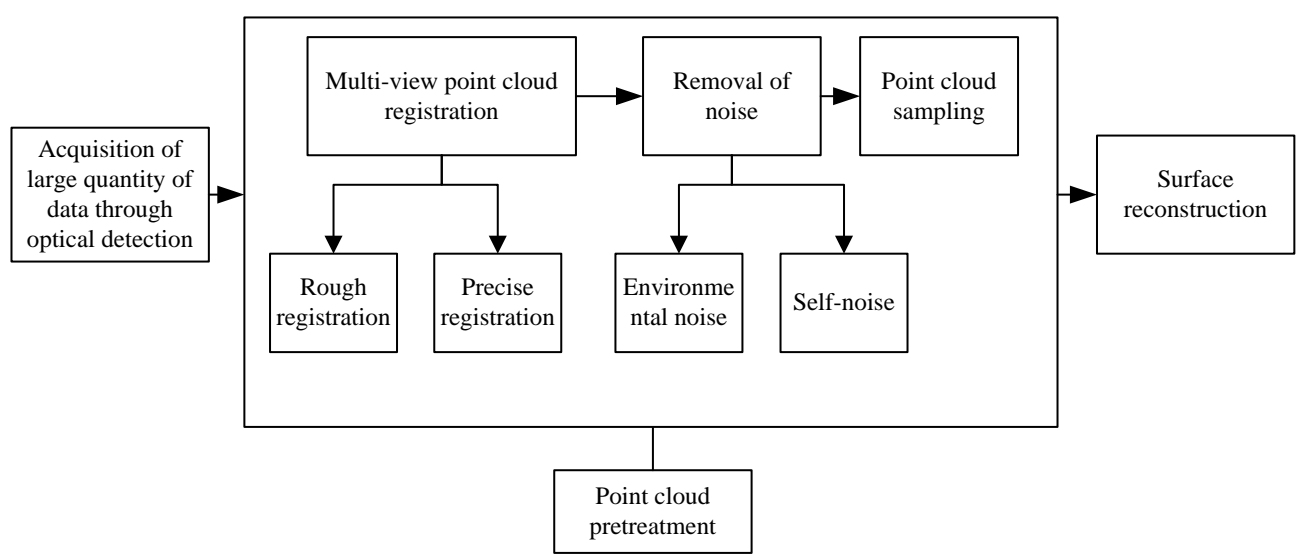

Fig. 1 Point Cloud Pretreatment Procedure

1) Multi-view point cloud registration

When three-dimensional optical surface scanning equipment collects data, objects shall be measured from several angles due to the restriction of scanning scope and orientation. Different angles correspond to different local coordinates and final results need to be unified into the world coordinate system. Multi-view point cloud registration is divided into rough registration and precise registration. As spatial geometric relationships between mark points keep constant in different views, rough registration uses this principle for rough positioning.

Precise registration adopts ICP algorithm and there is superposition among data measured in different angles. Mapping of the corresponding points shall be established according to the superposition and transformational matrix shall be figured out based on least squares algorithm and the given accuracy shall be calculated through iteration. In this way, data under different angles is converted to the same coordinate system.

2) Removal of noise

For optical non-contact measurement equipment, there are two sources of noise:

On the one hand, it is influenced by geometrical shape, structure of the measured work-piece, simplicity of surface color, strip arrangement, roughness and environmental lighting;

On the other hand, noise comes from measurement system error, such as resolution ratio of CCD camera, distortion error, computer processing error, manufacturing accuracy of mechanical equipment, calibrated error and scale-plate accuracy.

\section{Point cloud sampling}

Design principles of point cloud sampling

Point cloud sampling technology is an important step in point cloud pretreatment in the reverse engineering and it must meet 4 principles:

(1) It can process big data. Big data acquired from scanning contains tens of millions of points or hundreds of assistant point clouds generally and system must have the capacity to treat it;

(2) Speed shall be fast. As current market competition becomes more and more fierce, development period of new product shall be short and customer's treatment time for reverse modeling shall be as short as possible.

(3) Model precision shall be high. Digital analogy precision of point cloud fitting after sampling shall be as high as possible so as to meet requirement of the given precision;

(4) System robustness shall be good. With good robustness, system can treat point clouds in various complex conditions. In treatment of big data, program shall not crash or shut down.

3.2 Final objectives of point cloud sampling

The final evaluation objectives for good point cloud sampling algorithm are not reservation of many original points, fast treatment speed and few data point. The true criterion is that, the least points shall be used to represent the most geometrical characteristic information and treatment speed shall be the fastest and calculation time shall be the shortest on this basis.

Assume $P$ is the original point cloud, which corresponds to curved surface $S . P^{\prime}$ is the point 
cloud after sampling and it is constituted by ${ }^{n}$ points. Fitting surface definition is $S^{\prime}$. There is:

$$
\Delta\left(S, S^{\prime}\right)=S^{\prime}-S
$$

Where:

$\Delta\left(S, S^{\prime}\right)$-normal deviation of two curved surfaces;

$S^{\prime}$-fitting curved surface;

$S$ _CAD digital model of the existing product.

$\Delta\left(S, S^{\prime}\right)$ needs to meet Equation (2).

Where:

$$
\Delta\left(S, S^{\prime}\right) \leq E
$$

$E$-normal deviation of point cloud set according to customer's requirement.

Final objective of sampling is to meet requirement of Equation (2) and make ${ }^{n}$ as small as possible and point cloud gained after sampling is not entirely subset of the original point cloud.

Measurement index for point cloud sampling algorithm:

(1) High precision. Deviation between fitting surface of point cloud and ideal surface shall be within the allowable scope of precision after sampling and feature information of the original point cloud shall be reserved as many as possible;

(2) Fast speed. Sampling algorithm speed shall be as soon as possible. Updating speed of modern design product is accelerated and too much time in reverse engineering will bring much inconvenience to scientific research and enterprise and public institution. Even for excellent algorithm, if the calculation time is too much, its market application prospect is remote;

(3) Suitable simple degree. Data volume of point cloud after sampling; Sampling objective is to reduce data point of point cloud and data point shall be reduced as many as possible with guaranteed precision; too small point will also bring difficulty for follow-up modeling, therefore, suitable simple degree shall be selected according to the practical demand.

Algorithm can barely meet above 3 indexes in practical application and users shall consider them comprehensively during use. It is necessary to select suitable algorithm according to the application purpose. For judgment criterion for sampling termination, it must reach specified maximum permissible error or quantity of the specified points and it applies to occasions having high requirement on geometric accuracy of reconstruction.

\section{Application example}

The Thesis takes large scale hydraulic turbine blade of certain domestic steam turbine plant as research object. Photography measurement technology and surface scanning technology are combined for detection. Point cloud data of the blade is gained in the scanning and pretreatment is conducted. Data which undergoes treatment will be compared with CAD model of work-piece. There are more than 2 million points of original point clouds collected by blade model and there are 25000 points after level clustering method 1/80 is used for sampling. Compared with hundreds of points collected in coordinate measuring machine, it can meet requirement of measurement point quantity.

Three-dimensional detection and point cloud treatment process are shown in Fig.2. Fig. 2 (a) represents photography measurement of blade; Fig. 2 (b) is point cloud collected in surface scanning technology; Fig. 2 (c) is the final single breadth point cloud collected after point cloud pretreatment and sampling; Fig. 2 (d) is the goodness of fit between point cloud collected after Geomagic Qualify sampling and actual CAD digital model. Chromatogram map shows that point cloud treatment technology can meet requirement of engineering practice. 

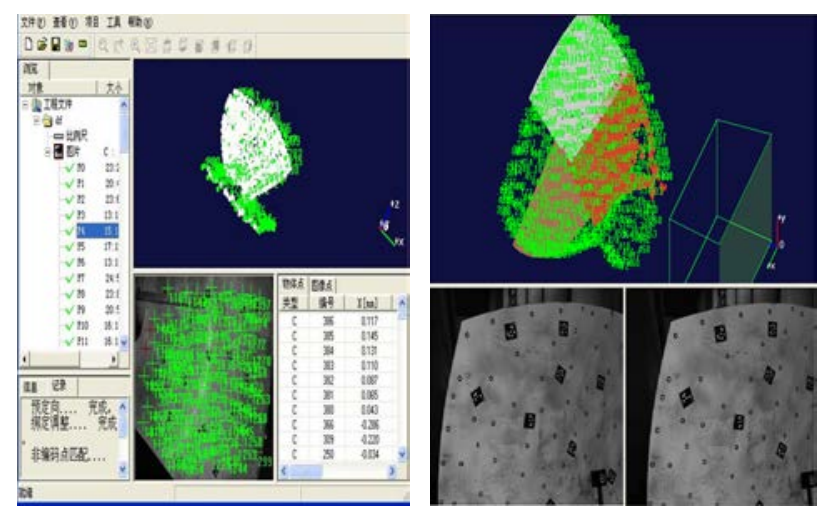

(a) Mark Point in Photography Measuremen

(b) Point Cloud in Surface Scanning
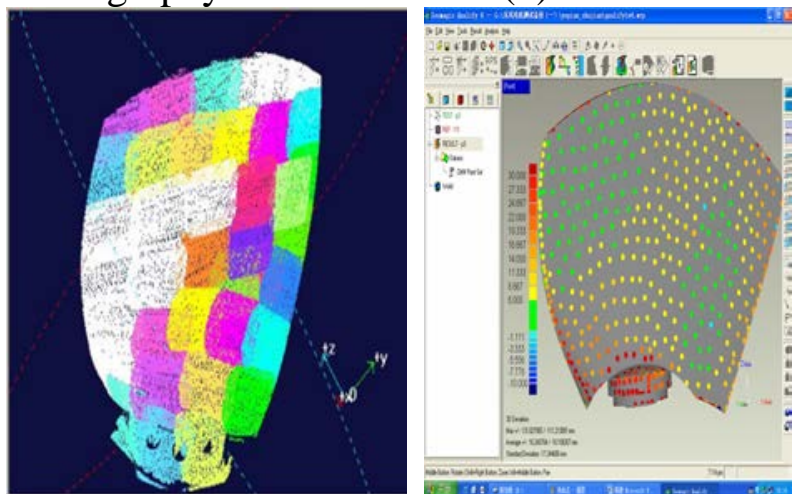

(c) Global Fitting of Point Cloud (d) Geomaogic Qualify Treats Point Cloud and Digital Model

Fig.2 Point Cloud Treatment in Three-dimensional Detection Process of Hydraulic Turbine Blade

\section{Conclusion}

The Thesis studies pretreatment technology and work flow of large quantity of point clouds gained in optical detection and proposes final judgment criterion $\Delta\left(S, S^{\prime}\right)$ during point cloud sampling. The judgment criterion meets requirement of high precision, fast speed and suitable simple principles and it can also reach the given maximum permissible error or quantity of the specified points and it applies to occasions having high requirement on geometric accuracy of reconstruction. The Thesis takes certain large scale hydraulic turbine blade as an example and conducts sampling and reverse design modeling accuracy analysis and proves validity and feasibility of three-dimensional optical detection point cloud sampling technology.

\section{Reference}

[1] Yang X H, Zhang Q J, Zhao D D, et al. UAV image automatic mosaic method based on matching of feature points[C]// Chinese Automation Congress. IEEE, 2013:33-36.

[2] An J, Liu G. Image mosaic algorithm based on feature points matching and automatic transform parameters identifying[J]. Infrared \& Laser Engineering, 2011, 40(3):564-569.

[3] Liu Y S, Paul J C, Yong J H, et al. Automatic least-squares projection of points onto point clouds with applications in reverse engineering[J]. Computer-Aided Design, 2006, 38(12):1251-1263.

[4] Bifari E N, Elrefaei L A. Automated Fingerprint Identification System based on weighted feature points matching algorithm[C]// International Conference on Advances in Computing, Communications and Informatics. IEEE, 2014:2212-2217.

[5] Quanqing L I, Ming L I. Research on Automatic Modeling Technology Based on Solid Feature for Reverse Engineering[C]// international conference on mechanical engineering and mechanics 2007. 2007. 